\title{
Influence of a Backward-Facing Step on Swept-Wing Boundary-Layer Transition
}

\author{
Jenna L. Eppink* \\ NASA Langley Research Center, Hampton, Virginia 23681 \\ Richard W. Wlezien亡 \\ Missouri University of Science and Technology, Rolla, Missouri 65409 \\ and \\ Rudolph A. King* and Meelan Choudhari \\ NASA Langley Research Center, Hampton, Virginia 23681
}

DOI: $\underline{10.2514 / 1 . J 057437}$

\begin{abstract}
Experimental measurements were performed on a swept flat-plate model with an airfoil leading edge and imposed chordwise pressure gradient to determine the effects of a backward-facing step on transition in a low-speed stationary crossflow-dominated boundary layer. Detailed hot-wire measurements were performed for three step heights ranging from 36 to $49 \%$ of the boundary-layer thickness at the step and corresponding to subcritical, nearly critical, and critical cases. In general, the step had a small localized effect on the growth of the stationary crossflow vortex, whereas the unsteady disturbance amplitudes increased with increasing step height. Intermittent spikes in instantaneous velocity began to appear for the two larger step heights. A physical explanation was provided for the mechanism leading to transition and the sudden movement in the transition front due to the critical steps. The large localized velocity spikes, which ultimately led to an intermittent breakdown of the boundary layer, were the result of nonlinear interactions of the different types of unsteady instabilities with each other and with the stationary crossflow vortices. Thus, the unsteady disturbances played the most important role in transition, but the stationary crossflow vortices also had a significant role via the modulation and the increased amplitude of the unsteady disturbances.
\end{abstract}

\begin{tabular}{|c|c|c|}
\hline \multicolumn{3}{|r|}{ Nomenclature } \\
\hline$C_{p}$ & $=$ & pressure coefficient; $\left(p-p_{\infty}\right) /(1 / 2) \rho U_{\infty}^{2}$ \\
\hline$c^{p}$ & $=$ & chord length, $\mathrm{m}$ \\
\hline$f$ & $=$ & frequency, $\mathrm{Hz}$ \\
\hline$H$ & $=$ & $\begin{array}{l}\text { high-frequency band (with } f \text { equal to } 800 \text { to } \\
1500 \mathrm{~Hz} \text { ) of disturbances measured behind the step }\end{array}$ \\
\hline$h$ & $=$ & step height, mm \\
\hline$L$ & $=$ & $\begin{array}{l}\text { low-frequency band (with } f \text { equal to } 80 \text { to } 200 \mathrm{~Hz} \text { ) } \\
\text { of disturbances measured behind the step }\end{array}$ \\
\hline$M$ & $=$ & $\begin{array}{l}\text { mid-frequency band (with } f \text { equal to } 200 \text { to } 800 \mathrm{~Hz} \text { ) } \\
\text { of disturbances measured behind the step }\end{array}$ \\
\hline$N$ & $=$ & $N$ factor; integrated disturbance growth rate \\
\hline$R e_{k}$ & $=$ & $\begin{array}{l}\text { Reynolds number based on excrescence height and } \\
\text { velocity at the excrescence height; } u_{k} h / \nu\end{array}$ \\
\hline$R e^{\prime}$ & $=$ & unit Reynolds number; $U_{\infty} / \nu, \mathrm{m}^{-1}$ \\
\hline$T u$ & $=$ & normalized turbulence \\
\hline$U$ & $=$ & $\begin{array}{l}1 / U_{\infty} \sqrt{(1 / 3)\left(u_{\mathrm{rms}}^{\prime 2}+v_{\mathrm{rms}}^{\prime 2}+w_{\mathrm{rms}}^{\prime 2}\right)} \\
\text { mean boundary-layer velocity in the } \\
\text { direction, } \mathrm{m} / \mathrm{s}\end{array}$ \\
\hline$U_{e}$ & $=$ & boundary-layer edge velocity, $\mathrm{m} / \mathrm{s}$ \\
\hline$U_{\infty}$ & $=$ & freestream velocity, $\mathrm{m} / \mathrm{s}$ \\
\hline$U_{\mathrm{rms}}^{\prime}$ & $=$ & $\begin{array}{l}\text { spanwise root mean square of steady disturbance } \\
\text { velocity } U^{\prime}, \mathrm{m} / \mathrm{s}\end{array}$ \\
\hline$U^{\prime}$ & $=$ & steady disturbance velocity, m/s \\
\hline
\end{tabular}

Presented as Paper 2015-2776 at the AIAA 45th AIAA Fluid Dynamics Conference, Dallas, TX, 22-26 June 2015; received 24 April 2018; revision received 25 July 2018; accepted for publication 11 September 2018; published online 21 November 2018. This material is declared a work of the U.S. Government and is not subject to copyright protection in the United States. All requests for copying and permission to reprint should be submitted to CCC at www.copyright.com; employ the ISSN 0001-1452 (print) or 1533-385X (online) to initiate your request. See also AIAA Rights and Permissions www.aiaa.org/randp.

*Research Aerospace Engineer, Flow Physics and Control Branch, M.S. 170. Member AIAA.

${ }^{\dagger}$ Vice Provost and Dean, College of Engineering and Computing, 305 McNutt. Fellow AIAA

${ }^{\ddagger}$ Research Aerospace Engineer, Computational Aerosciences Branch, M.S. 128. Associate Fellow AIAA.

\begin{tabular}{|c|c|c|}
\hline$v, w$ & $=$ & $\begin{array}{l}\text { velocity components in the } x, y \text {, and } z_{n} \\
\text { directions, } \mathrm{m} / \mathrm{s}\end{array}$ \\
\hline$u_{\mathrm{rms}}^{\prime}$ & $=$ & temporal root mean square of $u^{\prime}, \mathrm{m} / \mathrm{s}$ \\
\hline$u^{\prime}, v^{\prime}, w^{\prime}$ & $=$ & fluctuating components of velocity, m/s \\
\hline$x$ & $=$ & streamwise direction, $\mathrm{m}$ \\
\hline$x_{c}$ & $=$ & direction normal to the leading edge, $\mathrm{m}$ \\
\hline$x_{h}$ & $=$ & streamwise location of step, normalized by c \\
\hline$x_{r}$ & $=$ & $\begin{array}{l}\text { reattachment location in terms of the number of } \\
\text { step heights downstream of the step }\end{array}$ \\
\hline$x_{\mathrm{sh}}$ & $=$ & number of step heights downstream of step \\
\hline$y$ & $=$ & wall-normal direction, $\mathrm{mm}$ \\
\hline$z$ & $=$ & spanwise direction (parallel to the leading edge), $\mathrm{m}$ \\
\hline & $=$ & direction normal to side wall, $\mathrm{mm}$ \\
\hline$\Delta N$ & $=$ & shift in $N$ factor due to the excrescence \\
\hline$\delta$ & $=$ & boundary-layer thickness, $\mathrm{mm}$ \\
\hline$\lambda_{z}$ & $=$ & spanwise wavelength, $\mathrm{mm}$ \\
\hline
\end{tabular}

\section{Introduction}

$\mathbf{M}$ AINTAINING laminar flow over an extended portion of an aircraft wing surface can result in significant fuel savings due to the reduced skin-friction drag of a laminar boundary layer. However, this is a difficult objective because of the sensitivity of laminar boundary layers to surface irregularities on operational aircraft surfaces, which may arise either as a result of unavoidable manufacturing defects such as steps and gaps or due to structural fasteners in the form of screws and rivets. To avoid a loss of laminar flow due to such irregularities, aerodynamic designers must be able to specify proper manufacturing tolerances. This, in turn, requires an indepth understanding of how the various types of irregularities influence the transition process, including their interactions with the different types of boundary-layer instabilities that are responsible for transition.

Historically, the effects of two-dimensional (2-D) and threedimensional (3-D) excrescences on transition in 2-D boundary layers have been predicted using an empirical method known as the $R e_{k}$ criterion [1-5]. Here, $R e_{k}$ is defined as the Reynolds number based on the height of the excrescence and the undisturbed streamwise velocity at this roughness height. The $R e_{k}$ criterion correlates the measured onset of transition with a critical value of $R e_{k}$, such that the transition location first begins to move upstream from the transition 
location over a smooth surface as $R e_{k}$ increases above this critical value. Below this critical Reynolds number, the transition location is generally unaffected by the presence of the roughness. More recently, experimental and numerical studies have been performed to look at the effects of 2-D surface excrescences on the stability of 2-D boundary-layer disturbances [6-8]. For this method, it is assumed that a localized perturbation of the pressure distribution, caused by the surface imperfection, will cause a localized perturbation of the amplification characteristics of linear instability modes [9]. Note that this approach is primarily concerned with the effect of the surface imperfection on linear stability; thus, any effect of the imperfection on localized receptivity is neglected. Downstream of this perturbation, the amplification curve will become parallel to the nominal amplification curve. Hence, a linear stability theory is applied to determine a $\Delta N$; that is, the change in the logarithmic amplification ratio (i.e., the so-called $N$ factor) of the instabilities due to the presence of the excrescence. The majority of the work described previously has focused on twodimensional excrescences in two-dimensional Tollmien-Schlichting (TS)-dominated boundary layers.

There are considerably less data available for the effect of 2-D excrescences in 3-D boundary layers. Perraud and Seraudie [8] performed a systematic experimental study of 2-D steps on a swept wing with multiple sweep angles. For the larger (i.e., more negative) angle of attack (which resulted in a more favorable pressure gradient), the increased sweep caused the boundary layer to become more sensitive to the forward-facing step height (i.e., transition occurred for lower step heights as the sweep was increased). The results for a backward-facing step showed a similar trend, although the critical roughness height Reynolds numbers were even lower. Recently, Duncan et al. [10] performed flight experiments to determine the effect of 2-D steps on transition over a swept wing. Their results indicated that the addition of the crossflow instability caused the transition location to move forward relative to the two-dimensional boundary-layer (i.e., unswept) case, which was in agreement with the results of Perraud and Seraudie [8]. In the companion wind-tunnel testing performed by Duncan et al. [11], the results indicated that the 3-D (i.e., swept wing) boundary layer was slightly more sensitive to 2-D forward-facing steps than the corresponding 2-D (i.e., zero sweep) boundary layer, but a similar sensitivity was not observed for 2-D backward-facing steps. Duncan et al. [10] also performed hotwire measurements downstream of the step for forward- and backward-facing steps to determine the effect of the steps on stationary crossflow instabilities. They found that the steps caused an increase in $N$ factor for the stationary crossflow disturbances; however, the amplitudes of the stationary crossflow disturbances were very low at the step, and therefore the uncertainty in amplitude measurement was high. Tufts et al. [12] performed computations for the same case and found that the backward-facing step did not amplify the stationary crossflow modes. They also verified the existence of a traveling instability in the recirculating region downstream of the step. For the same model, Crawford et al. [13] examined the effect of step location on transition on a swept wing for two step locations in flight and in wind-tunnel tests. They found that the step at $1 \% x / c$ behaved differently in flight than in the wind tunnel, presumably because it was near the neutral point in flight, but upstream of the neutral point in the wind tunnel. They concluded that the step could act as a receptivity source for stationary crossflow vortices if it was near the neutral point.

Eppink et al. [14] acquired detailed hot-wire measurements downstream of a $2-\overline{\mathrm{D}}$ backward-facing step in a stationary crossflowdominated flow on a swept flat plate with a pressure body. The results indicated a local increase in stationary crossflow amplitude downstream of the step. Detailed measurements of the unsteady instabilities downstream of the step were presented, and it was determined that the effect of the stationary crossflow instability on transition was seen through the modulation of the unsteady instabilities. Additionally, three distinct families of unsteady disturbances were identified downstream of the step, corresponding to a traveling crossflow-type disturbance, a Tollmien-Schlichting instability, and a shear-layer instability. The same case was further studied by Eppink and Yao $[15,16]$ using particle image velocimetry, revealing more details of the instabilities and the breakdown of the flow. Balakumar et al. [17] recently performed linear parabolized stability equation and direct numerical simulation computations for a supersonic swept-wing case to study the effect of 2-D excrescences on stationary crossflow. Their results indicated a linear effect on the growth of the stationary crossflow modes, but the different modes were affected differently. The shorter wavelength modes were amplified due to the step, but the longer wavelength modes were actually stabilized relative to the baseline case.

There is still more knowledge to be gained concerning the details of how transition is affected on a swept wing when two-dimensional excrescences are introduced. The earlier research in this area was concerned primarily with studying the effect of forward- and backward-facing steps on the transition location. Before the current experiment and follow-on work, very little data existed that could provide an understanding of how the steps actually affected the boundary-layer instabilities and how this ultimately led to transition. To gain more insight into this problem, an experiment was performed to enable a detailed look at the effect of a backward-facing step on transition in an otherwise stationary crossflow-dominated swept-wing flow. Results were presented previously by Eppink et al. [14] for a single height of the backward-facing step. The current work includes the effect of step height on the disturbance growth and focuses on characterizing the mechanisms leading to transition. Boundary-layer measurements are presented for three different step heights.

\section{Experimental Setup}

The experiment was performed in the $2 \mathrm{ft}$ by $3 \mathrm{ft}$ low-speed boundary-layer channel at the NASA Langley Research Center. The tunnel is a closed-circuit facility with a $0.61-\mathrm{m}$-high by $0.91-\mathrm{m}$-wide by $6.1-\mathrm{m}$-long test section. The tunnel can reach speeds of up to $45 \mathrm{~m} / \mathrm{s}\left(R e^{\prime}=2.87 \times 10^{6} / \mathrm{m}\right)$ in the test section. Freestream turbulence intensity levels

$$
T u=\frac{1}{U_{\infty}} \sqrt{\frac{1}{3}\left(u_{\mathrm{rms}}^{\prime 2}+v_{\mathrm{rms}}^{\prime 2}+w_{\mathrm{rms}}^{\prime 2}\right)}
$$

were measured using a crosswire in an empty test section; they were found to be less than $0.06 \%$ for the entire speed range of the tunnel and less than $0.05 \%$ at the test speed of $26.5 \mathrm{~m} / \mathrm{s}$. This value represented the total energy across the spectrum $(0.25 \mathrm{~Hz}$ to $10 \mathrm{kHz})$, and it has not been filtered to remove the low-frequency acoustic component. Based on the criteria outlined by Saric and Reshotko [18], this tunnel is considered a low-disturbance facility for the purposes of conducting transition experiments.

The 0.0127-m-thick flat-plate model consists of a 0.41-m-long leading-edge piece swept at $30 \mathrm{deg}$ and the larger flat-plate piece downstream (see Fig. 1). The model is $0.91 \mathrm{~m}$ wide (thus spanning the width of the test section) and $2.54 \mathrm{~m}$ long on the longest (i.e., inboard) edge. The leading-edge and flat-plate pieces can be adjusted relative to each other using precision shims to create either forward-facing or backward-facing 2-D steps of different heights that are parallel to the leading edge. The leading-edge piece is polished to a surface finish of $0.2 \mu \mathrm{m}$, and the larger downstream plate has a surface finish of $0.4 \mu \mathrm{m}$. The surface finish was measured using a contact profilometer. The chord $c$ is taken as the longest edge of the

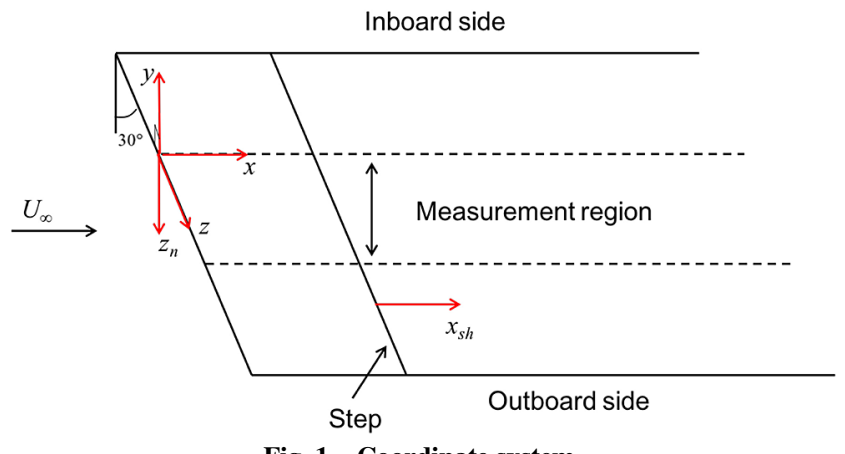

Fig. 1 Coordinate system. 
plate $(2.54 \mathrm{~m})$ and is used to nondimensionalize quantities throughout the paper. Thus, the step is located at $x / c=0.161$. A leading-edge contour is designed for the bottom side of the plate to make the suction peak less severe, thereby avoiding boundary-layer separation over the bottom portion of the leading edge that will result in unsteady meandering of the attachment line.

A 3-D pressure body along the ceiling was designed to induce a streamwise pressure gradient so that transition would be dominated by stationary crossflow. The ceiling liner was contoured to simulate nearly infinite swept-wing flow within a midspan measurement region with a width of $0.3 \mathrm{~m}$. This was achieved by designing the liner such that the $C_{p}$ contours were parallel to the leading edge within the core region. The ceiling liner was fabricated out of hard foam by using a computer-controlled milling machine.

All measurements were performed at a freestream velocity of $26.5 \mathrm{~m} / \mathrm{s}\left(R e^{\prime}=1.69 \times 10^{6} / \mathrm{m}\right)$. The data were acquired by using a hot wire mounted on a traversing system that could be moved in all three $\left(x, y\right.$, and $\left.z_{n}\right)$ directions. Detailed boundary-layer measurements allowed for tracking of the instability growth and determining the effect of the backward-facing step on the various flow instabilities. Additionally, sublimating chemical flow visualization was performed using naphthalene to determine the transition location. To vary the amplitude of the stationary crossflow disturbance approaching the step, a periodic array of discrete roughness elements (DREs) with known dimensions and spacing was mounted just aft of the leading edge, near the neutral location $(x=50 \mathrm{~mm})$. Overall, three different leading-edge roughness configurations were investigated: a clean leading edge, and two DRE configurations. Both DRE configurations involved a spanwise spacing $\lambda_{z}$ of $11 \mathrm{~mm}$ and a roughness height of approximately $20 \mu \mathrm{m}$. They differed in the diameter of the circular planform DREs, with the small DRE configuration involving a planform diameter of $2.75 \mathrm{~mm}$ and the large DRE configuration having a diameter of $4.4 \mathrm{~mm}$. The large-diameter DREs led to larger initial amplitudes of the stationary crossflow disturbance due to the enhanced receptivity. The spacing of the DREs $(11 \mathrm{~mm})$ corresponded to the most amplified stationary crossflow wavelength calculated for the baseline case with no step.

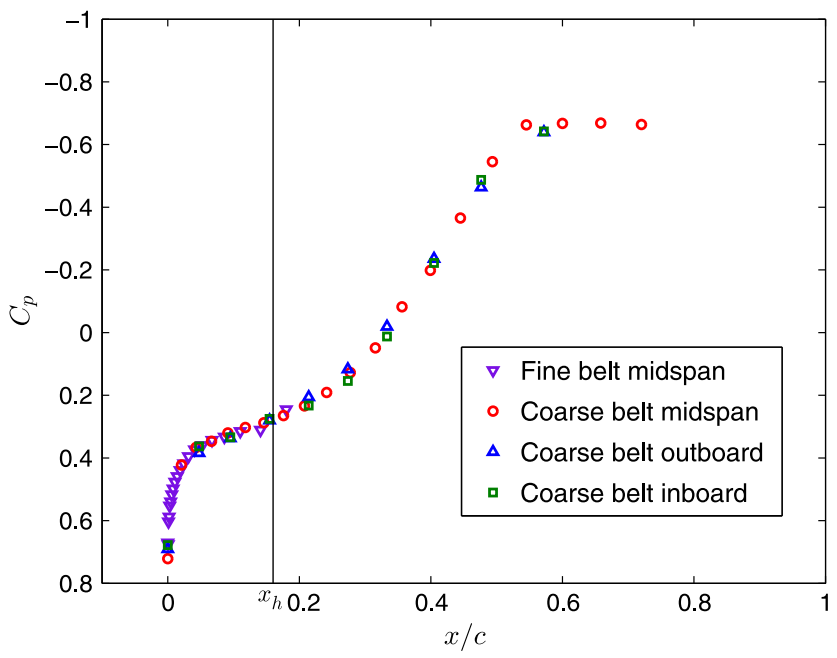

Fig. 2 Surface pressure distribution with all four pressure belts.
Hot-wire data were obtained using a constant-temperature anemometer with a $1: 1$ bridge configuration. Single-sensor hot-wire probes were used throughout the test for the boundary-layer measurements. The wires were $5-\mu \mathrm{m}$-diam platinum-plated tungsten wires with a sensor length of $1.25 \mathrm{~mm}$. The data were ac coupled at $0.25 \mathrm{~Hz}$ to acquire the fluctuating velocity component and low-pass filtered at a cutoff of $10 \mathrm{kHz}$. Data were typically digitized at a sampling rate of $25 \mathrm{kHz}$. For more details of the experiment setup, as well as the data reduction and uncertainty analysis, refer to the work of Eppink et al. [14].

The streamwise pressure distribution along the model is shown in Fig. 2 for the no-step case. These measurements were performed using a series of pressure belts (see the work of Eppink et al. [14] for more details). A comparison of the pressure distributions obtained using the various belts verifies very good spanwise uniformity across the measurement region.

\section{Results and Discussion}

Detailed boundary-layer measurements were acquired for three heights of the backward-facing step (BFS) $(h / \delta \approx 0.36,0.45$, and $0.49)$ and are presented in this section. The two larger step heights bracketed the critical step height, which was defined as the step height above which transition abruptly moved upstream. The $h / \delta \approx 0.45$ step height was slightly lower than the critical step height, whereas the $h / \delta \approx 0.49$ step height was slightly larger than the critical step height. Studying transition at these nearly critical step heights was expected to provide insight into the dominant transition mechanism (s). The smallest step height of $h / \delta \approx 0.36$ was studied briefly to characterize the disturbance evolution at a significant, yet strongly subcritical step height. For this subcritical case, only one leadingedge roughness configuration was tested (small DREs). More detailed results for the baseline and largest step height cases were presented by Eppink et al. [14], but a few of those results are used herein for comparison purposes.

Figure 3 shows the mean boundary-layer profiles at numerous streamwise stations for all three step heights with small DREs, along with computations from the baseline case. These computations were performed using the experimental midspan $C_{p}$ distribution from the baseline case, under the assumption of an infinite span swept airfoil. See the work of Eppink et al. [19] for more details of the computation. The experimental profiles plotted are the absolute value of the streamwise velocity $|u|$ because a single hot wire cannot be used to determine flow direction.

The shape of the profiles reveals flow separation downstream of the step. For the profiles just downstream of the step, it is expected that the streamwise velocity near the wall is negative because there is no reason for the velocity to increase near the wall. Based on the shape of the profiles near the wall, reattachment occurs by approximately 30 step heights, and the profiles return to the unperturbed state by approximately 50-55 step heights downstream of the step. Similar results are obtained for all three of the step heights that are studied. Overall, the experimental results match well with the boundary-layer profiles computed using the measured $C_{p}$ distribution in the midspan region far downstream of the step, although there is some discrepancy near the wall for some of the profiles. The discrepancy could be caused by the distortion of the profiles due to the stationary crossflow (because the experimental profiles are taken at a single spanwise

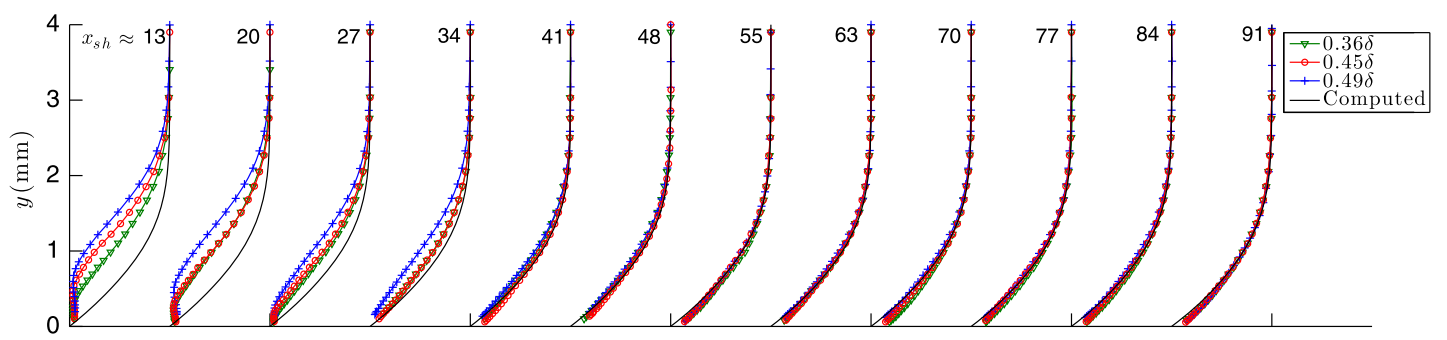

Fig. 3 Measured $|u|$ profiles (symbols) for all three step heights with small DREs and their comparison with computed profiles for the baseline case (solid lines). 
Table 1 Backward-facing step heights and approximate transition locations for no-DRE cases $(\delta=2.4 \mathrm{~mm})$

\begin{tabular}{lccc}
\hline \hline Case & $\begin{array}{c}\text { Step } \\
\text { height }(h / \delta)\end{array}$ & $\begin{array}{c}\text { Transition } \\
\text { location }(x / c)\end{array}$ & $\begin{array}{c}\text { Transition } \\
\text { location }\left(x_{\text {sh }}\right)\end{array}$ \\
\hline Baseline & 0 & 0.48 & ${\mathrm{~N} / \mathrm{A}^{\mathrm{a}}}^{\text {Small }}$ \\
Intermediate & 0.36 & 0.48 & 929 \\
Large & 0.45 & 0.48 & 722 \\
\hline \hline
\end{tabular}

aN/A denotes "not applicable".

station). Additionally, the near-wall proximity of the hot wire can increase heating of the wire, resulting in higher measured velocities.

Approximate transition locations for each step height are tabulated in Table 1. These results are based on the naphthalene flow visualization results for the no-DRE case for each step height, shown in Fig. 4. The transition location is averaged over the outboard half of the measurement region because this is the region where the hot-wire measurements were performed. Results are given in terms of $x / c$ and in terms of $x_{\mathrm{sh}}$ for comparison with later results.

In the naphthalene images (Fig. 4), white regions indicate the presence of the naphthalene coating, and hence a laminar state of the boundary layer. Dark regions indicate areas of higher skin friction associated with nearly to fully turbulent flow. Note that the optical access of the camera is limited to $x / c \approx 0.48$. Thus, if the transition location extends past $x / c=0.48$, it is listed as 0.48 . For the baseline case and smaller step heights, transition occurs beyond $x / c=0.48$. In these images, the transition location is generally not visible on the outboard half of the measurement region due to a visual obstruction (thus, the top-right corner of the image is cropped out), but it is visible on the inboard side, occurring shortly downstream of $x / c=0.48$.

The most significant observation from Table 1 is the rapid upstream movement in transition location from $x / c^{-}=0.48$ for the intermediate step height $(h / \delta=0.45)$ to $x / c=0.27$ for the large step height $(h / \delta=0.49)$. In other words, a less than $9 \%$ increase in step height leads to a nearly $45 \%$ decrease in the laminar flow length. Conventionally, such rapid upstream movement in the transition onset location is associated with a bypass mode of transition, wherein the transition process bypasses the linear instabilities of the original unperturbed flow. Although the latter holds to some degree in the present case as well, it is only partly true, as revealed by the subsequent findings. For this reason, we choose not to label the transition mechanism as a case of bypass transition and focus instead on characterizing the details of this physical mechanism.

As mentioned previously, earlier measurements [14], which were focused on the largest step height, revealed that the BFS resulted in only a small localized increase in the growth of the stationary crossflow vortices, but it also introduced unsteady disturbances that were primarily responsible for transition. Nonetheless, the stationary crossflow vortices played a role in the transition process because they caused the unsteady instabilities to become highly modulated, leading to larger peak amplitudes and an earlier onset of transition.
Thus, the following sections will focus on characterizing the effect of step height on the growth of the stationary crossflow instability (Sec. III.A) and the effects of the step height (Sec. III.B) and the amplitude of the stationary crossflow vortex on the amplification of nonstationary disturbances (Sec. III.C). Understanding these individual aspects helps to clarify the respective roles of each of the instability types and ultimately leads to a characterization of the path to transition in the presence of a swept backward-facing step.

\section{A. Effect of Step Height on Stationary Crossflow Growth}

Contour plots of mean streamwise velocity are shown in Fig. 5 for the intermediate and large step heights for the small D̄RE configuration at two locations downstream of the step. These plots illustrate the mean flow modulation caused by the stationary crossflow, which is not strongly affected by the step height. However, one observes a reduced prominence of the $5.5 \mathrm{~mm}$ harmonic at the largest step height, as indicated by the spanwise distance between adjacent peaks of the contours in blue and yellow regions.

The effect of the step on the stationary crossflow vortices is illustrated further by examining the growth of the stationary crossflow instability for all configurations tested during the experiment. Results are shown in Fig. 6 for the primary $\left(\lambda_{z}=11 \mathrm{~mm}\right)$ mode and in Fig. 7 for the harmonic $\left(\lambda_{z}=5.5 \mathrm{~mm}\right)$ mode. The amplitudes are plotted along with the $N$-factor plots. The $U_{\text {rms }}^{\prime}$ values are calculated at each streamwise location by integrating the wavelength spectra of the steady streamwise velocity component at each wall-normal location over a range of wavelengths around the primary mode $\left(\lambda_{z}=8\right.$ to $\left.20 \mathrm{~mm}\right)$ and harmonic mode $\left(\lambda_{z}=3\right.$ to $\left.8 \mathrm{~mm}\right)$. The $U_{\mathrm{rms}}^{\prime}$ amplitudes plotted in Figs. 6 and 7 correspond to the peak value found at each streamwise location.

Hot-wire measurements for the baseline case with DREs began at $x / c \approx 0.12$, i.e., upstream of the step location. However, because the upstream influence of the step was expected to be limited to the immediate vicinity of the step, measurements with the backwardfacing step were mainly limited to the region downstream of the step. Thus, the disturbance amplitude at $x / c \approx 0.12$ from the baseline case with the specific type of DREs was also used as the initial (i.e., reference) amplitude for $N$-factor calculation for the step cases with the same types of DREs. The most upstream measurement location for the no-step no-DRE case was farther downstream at $x / c=0.18$, and hence the initial amplitude was chosen such that the $N$ factor at $x / c=0.18$ matched the $N$ factor for the small DRE case at this location, which was nearly equivalent to the $N$ factor for the large DRE case as well.

Measurements for the two smaller step heights were performed in phase 2 of the experimental campaign that involved a different batch of DREs than that used during the previous measurements involving the baseline case and the largest height step with $h / \delta \approx 0.49$. Unfortunately, this second batch of DREs was found to be approximately $16 \%$ thicker than the previous batch. Thus, it was expected that the initial amplitudes for the DRE cases with $h / \delta=0.36$

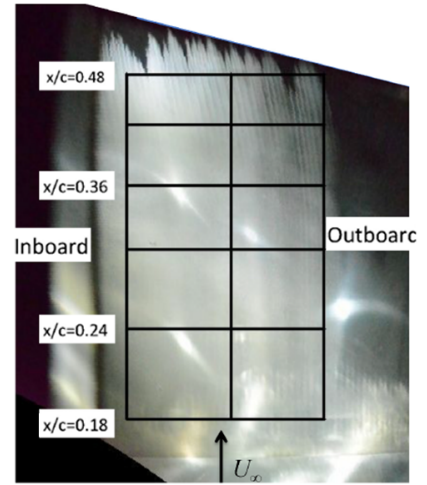

a) $h / \delta=0$

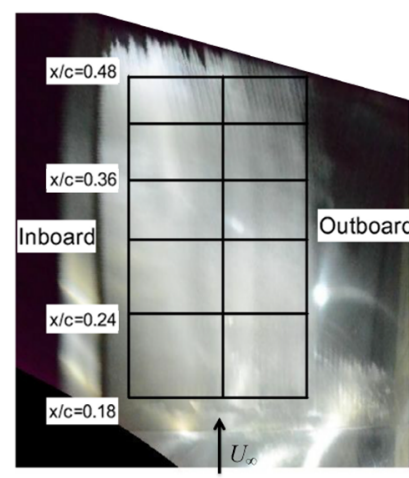

b) $h / \delta=0.36$

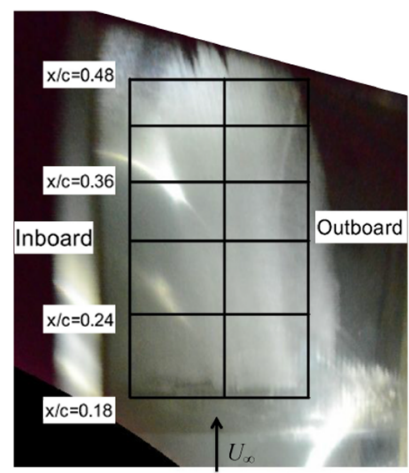

c) $h / \delta=0.45$

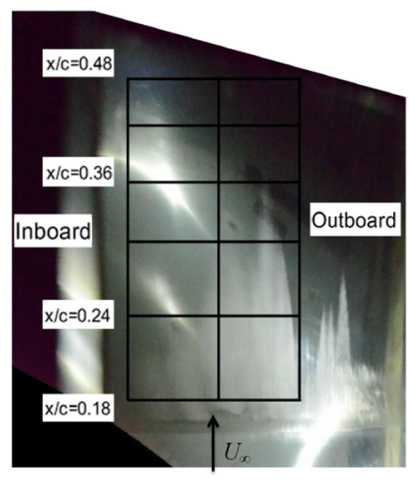

d) $h / \delta=0.49$

Fig. 4 Naphthalene flow visualization for all step heights. 


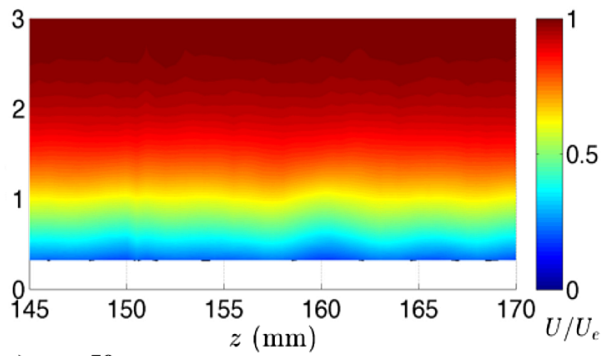

a) $x_{s h}=50$
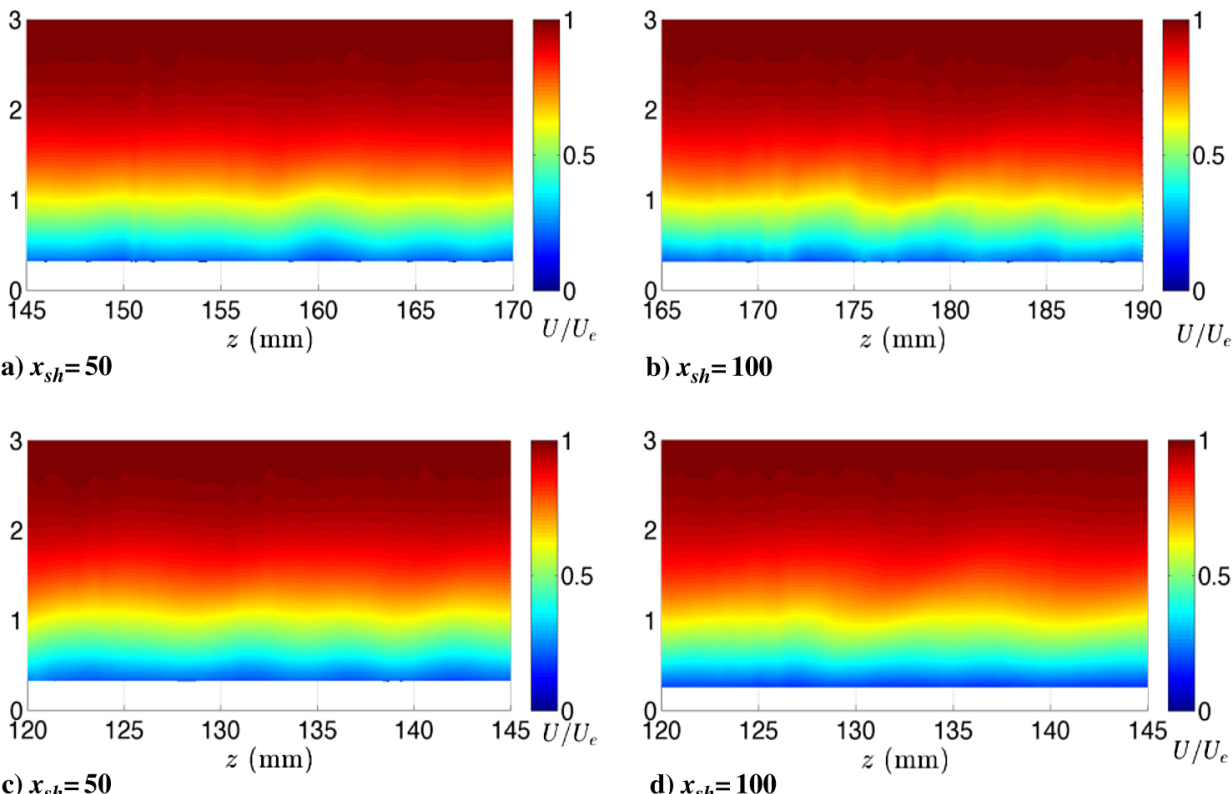

b) $x_{s h}=100$

Fig. 5 Contours of mean flow with small DREs for intermediate (top row) and large (bottom row) step heights.

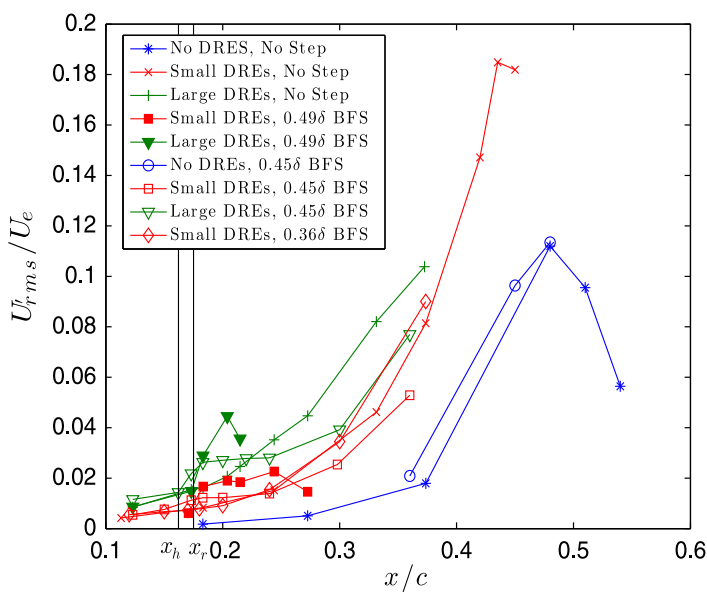

a) Amplitude evolution of stationary crossflow ( $\lambda_{z}=8$ to $20 \mathrm{~mm}$ )

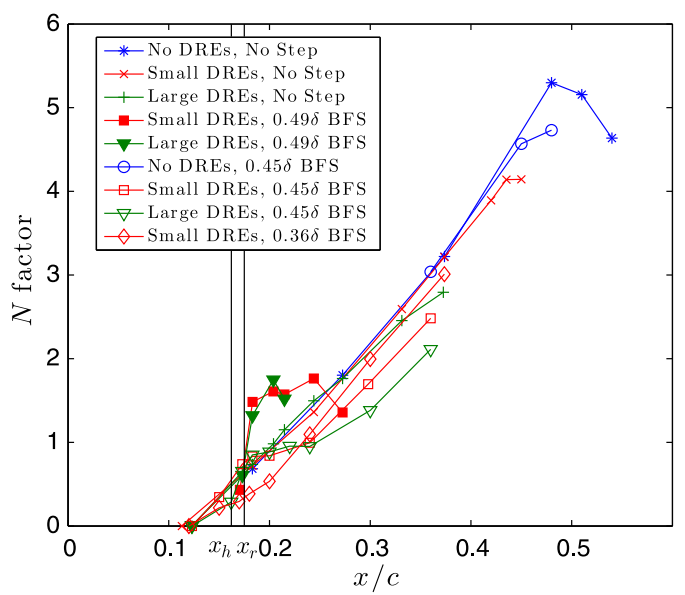

b) Growth of stationary crossflow mode with $\lambda_{z}=11 \mathrm{~mm}$

Fig. 6 Amplitudes and $N$ factors of primary stationary crossflow mode. Step $\left(x_{h}\right)$ and reattachment $\left(x_{r}\right)$ locations are indicated by the black vertical lines.

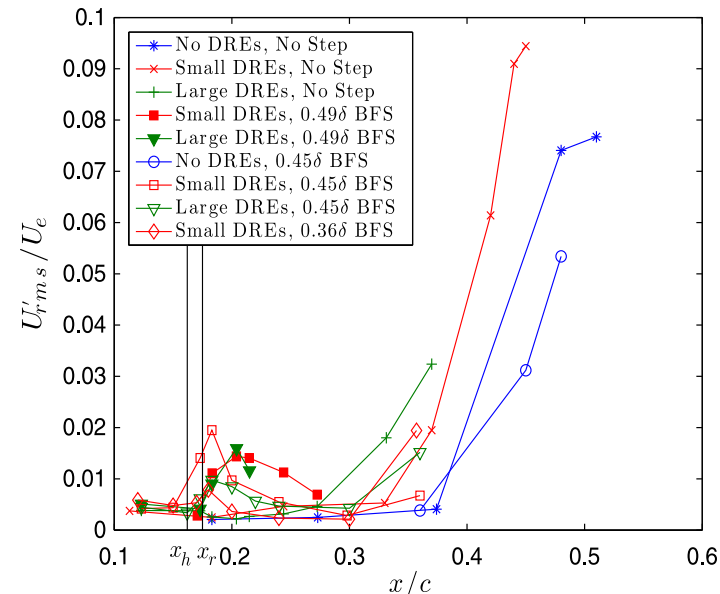

a) Amplitude evolution of stationary crossflow $\left(\lambda_{z}=3\right.$ to $8 \mathrm{~mm}$ )

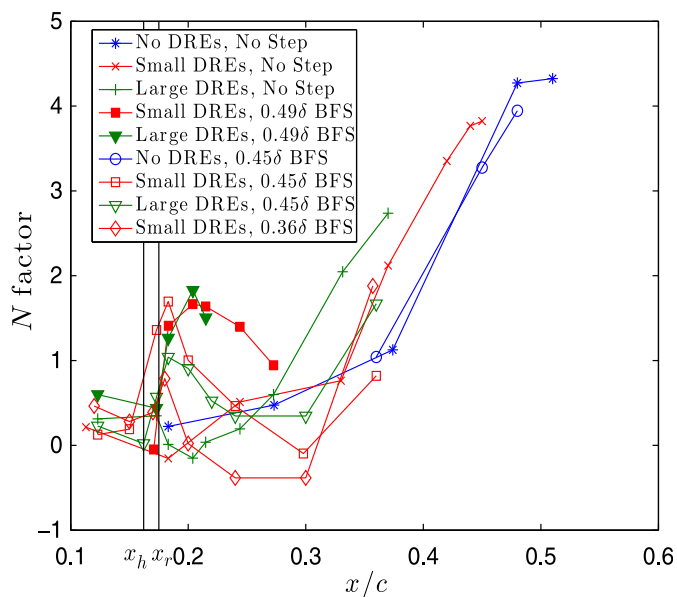

b) Growth of $5.5 \mathrm{~mm}$ stationary crossflow mode

Fig. 7 Amplitudes and $N$ factors of harmonic stationary crossflow mode. Step $\left(x_{h}\right)$ and reattachment $\left(x_{r}\right)$ locations are indicated by the black vertical lines. 
and 0.45 would be larger than those during the earlier measurements for the large step case. The amplitudes at $x / c=0.12$ were used as the initial amplitudes for all of the DRE cases for these two step heights. However, the initial measurement location for the no-DRE case with a step height of $h / \delta \approx 0.45$ was farther downstream at $x / c=0.36$, and so the $N$ factor at this location was matched with the $N$ factor for the no-DRE no-step case to determine the initial amplitude. The initial amplitudes were indeed found to be larger by approximately $25 \%$.

As seen in Fig. 6, the step has a local effect on the growth of the stationary crossflow, causing a local increase in amplitude near the step that increases with step height (compare lines of the same color near the step). The exception is the smallest step (red diamonds), which appears to cause a local decrease in amplitude of the primary mode. This decrease is more clearly evident in the $N$-factor plot (Fig. 6b). The $N$ factors near the step for the two DRE cases agree well with each other for each step height (compare filled and empty squares and triangles in Fig. 6b). No measurements were acquired near the step for the no-DRE case due to the small amplitude of the stationary crossflow at the step. The maximum $\Delta N$ values, calculated as the maximum deviation from the baseline $N$-factors, are $-0.465,0.113$, and 0.818 for the small, intermediate, and large step cases, respectively. The largest step causes a much larger $\Delta N$ for the primary mode that is over seven times the $\Delta N$ for the intermediate step case.

There appears to be a fairly significant reduction in stationary crossflow amplitude downstream of the step for the small and large DRE cases for the intermediate step height $(x / c>0.25)$ as compared to the baseline case. No such effect is seen for the no-DRE case. Balakumar et al. [17] observed a decrease in the linear growth of stationary crossflow downstream of a backward-facing step. However, the reduction in amplitude seen in the present work is probably not a linear effect but is likely due to the flow beginning to undergo breakdown. Transition occurs shortly downstream of the step for the large step height. The flow downstream of the intermediate step does not become fully turbulent, as indicated by the naphthalene flow visualization, until much farther downstream. However, as will be discussed in depth in the next section, the flow begins to undergo an intermittent breakdown starting shortly downstream of the step. The reduction in amplitude is probably due to the fact that breakdown extends over a very long region at this step height. It is likely that the breakdown process is accompanied by a decrease in the amplitude of the stationary crossflow disturbance.

The smallest step height was studied in order to determine whether this apparent reduction in stationary crossflow disturbance amplitude was a linear effect of the step or if it was an effect of the flow starting to undergo breakdown. As seen in Fig. 6, the small step apparently caused a local decrease in stationary crossflow amplitude close to the step, but the amplitude soon collapsed back to that of the baseline case. Thus, it appeared that the reduction in stationary crossflow amplitude far downstream of the step at the larger step heights was due to the flow beginning to undergo breakdown.
A local effect of the step is also seen for the harmonic stationary crossflow mode (Fig. 7). For the larger step heights, this harmonic mode increases abruptly downstream of the step but then decays. For the cases that did not transition near the step, the harmonic mode remains low near the step location but begins to increase significantly in amplitude farther downstream as the transition location is approached. For the most part, the local increase in amplitude near the step increases with increasing step height. One exception is the small DRE case for the $0.45 \delta$ BFS (red squares), which reaches larger amplitudes shortly downstream of the step relative to the small DRE case for the $0.49 \delta$ BFS (red filled squares). The maximum $\Delta N$ values near the step for the harmonic mode are approximately $0.56,1.47$, and 1.60 for the small, intermediate, and large step cases, respectively. These values are significantly higher than the $\Delta N$ values for the primary mode, particularly for the two larger step heights.

\section{B. Effect of Step Height on Unsteady Disturbance Growth and Breakdown}

Results for the largest step height were discussed in detail by Eppink et al. [14], but they will be summarized briefly here as context for the following discussion of the results for other step heights. The velocity spectra downstream of the step were rich with unsteady disturbances in a broad frequency band $(f \approx 80$ to $1500 \mathrm{~Hz})$. These unsteady disturbances were not present in the no-step case, and they are believed to be directly responsible for transition in the presence of the step because the amplitudes of stationary crossflow disturbances measured near the transition onset location were too low to initiate transition via their secondary instabilities (corresponding to $f>2000 \mathrm{~Hz}$ in the baseline case). Data were acquired simultaneously from two hot wires in the boundary layer to extract phase speed and wave angle information for the measured disturbances. The measurements revealed three different disturbance bands corresponding to a traveling crossflow type disturbance ( $f=80$ to $200 \mathrm{~Hz}$ ), a TS-like disturbance ( $f=200$ to $800 \mathrm{~Hz})$, and a shear-layer disturbance $(f=800$ to $1500 \mathrm{~Hz})$. These frequency bands will henceforth be referred to as the $\mathrm{L}$ band, the $\mathrm{M}$ band, and the $\mathrm{H}$ band for low, middle, and high, respectively. The unsteady disturbances were modulated in the spanwise direction by the stationary crossflow vortices, resulting in a pattern of alternating peaks and valleys in the amplitude distribution with a spanwise wavelength corresponding to the dominant stationary crossflow mode. The mean streamwise velocity and bandpass-filtered $u_{\mathrm{rms}}^{\prime}$ contours are shown in Fig. 8 for the two large step height cases with small DREs applied. The amplitude modulation of the unsteady instabilities is evident in these figures. The peak amplitudes in each frequency band increase with step height. The modulation appears to have a similar character between the two step heights, although the $\mathrm{M}$ band does appear somewhat different in character. For clarity, the discussion in the remainder of this section will focus on the small DRE case for all three nonzero step heights.

The streamwise evolution of disturbances from each of the three frequency bands was studied by choosing a spanwise peak location of

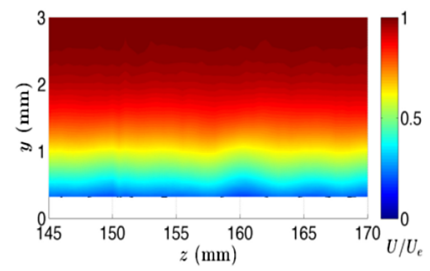

a) Mean flow

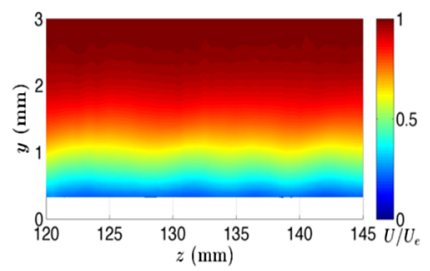

e) Mean flow

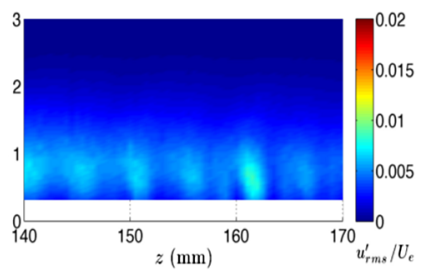

b) $\mathrm{L}$ band

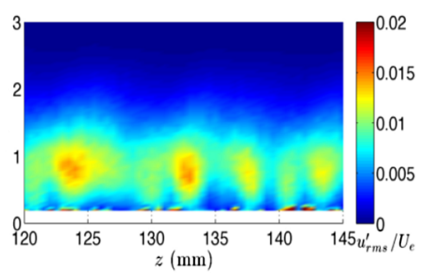

f) $L$ band

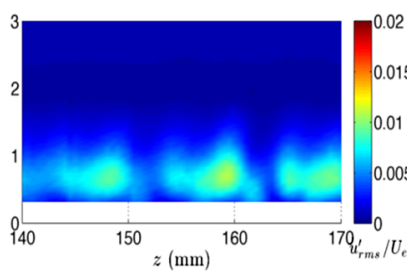

c) M band

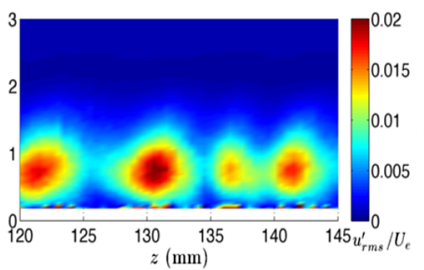

g) $\mathrm{M}$ band

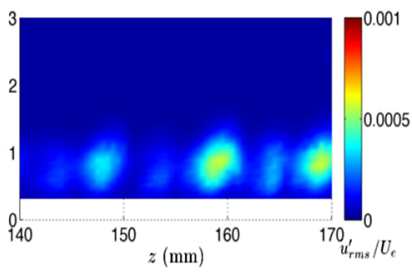

d) $\mathbf{H}$ band

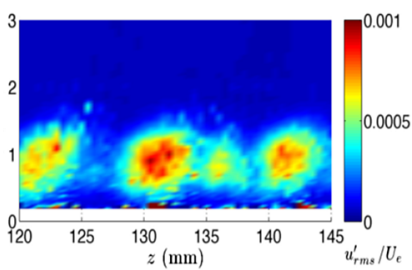

h) $\mathbf{H}$ band

Fig. 8 Contours of mean flow and rms velocity for small DREs at $x_{\mathrm{sh}} \approx \mathbf{5 0}$ for intermediate (top row) and large (bottom row) step heights. 
the $u_{\mathrm{rms}}^{\prime}$ amplitude [for instance, $(y, z) \approx(0.7,132) \mathrm{mm}$ in Fig. $8 \mathrm{~g}$ ] and tracking that peak location downstream. A single boundary-layer profile in $y$ was acquired at the spanwise location of this peak for numerous streamwise stations. The peak location was based on the amplitude distribution for the $\mathrm{M}$ band because the modulation patterns of the $\mathrm{M}$ and $\mathrm{H}$ bands were similar, and thus the spanwise peak locations were also close to each other. The L-band distortion was somewhat different in comparison with the $\mathrm{M}$ and $\mathrm{H}$ bands, and the peak spanwise location was typically offset from the peak of the $\mathrm{M}$ band by a few millimeters. Therefore, although the measured amplitudes were probably not the absolute peak of the L-band disturbance, the observed trends should still hold. Throughout the boundary-layer scans, spanwise scans were performed to verify that the peak location of the $\mathrm{M}$ band was tracked accurately.

The streamwise evolution of the unsteady disturbance amplitudes derived from this approach is shown in Fig. 9. The disturbance amplitudes in this figure correspond to the maximum of the wallnormal profile of $u_{\mathrm{rms}}^{\prime}$ at that streamwise location. Note that the $y$ axis is different for the H-band plot (Fig. 9c). There is some growth evident in all frequency bands for the two largest step heights. All three disturbance bands grow quickly, starting just downstream of the step for the largest step height (blue lines). Below the critical step

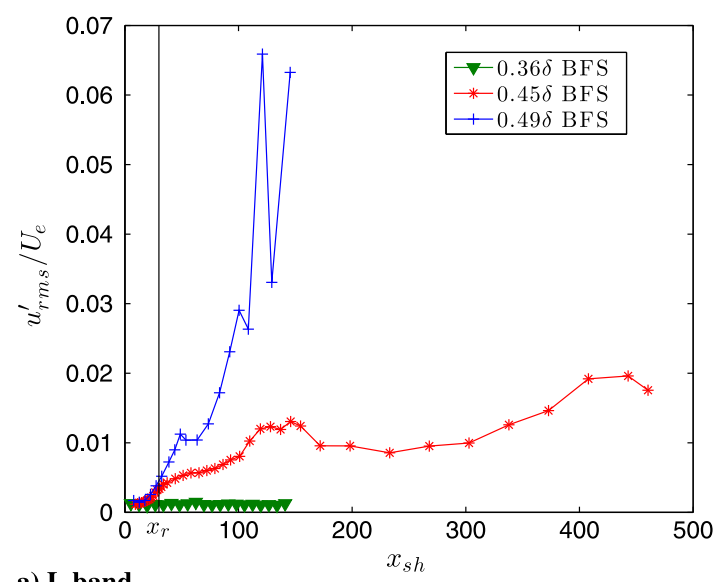

a) $L$ band

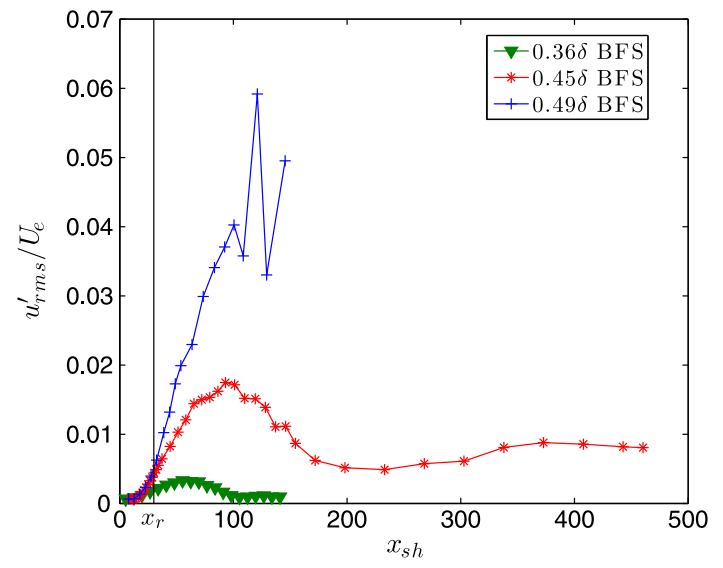

b) M band

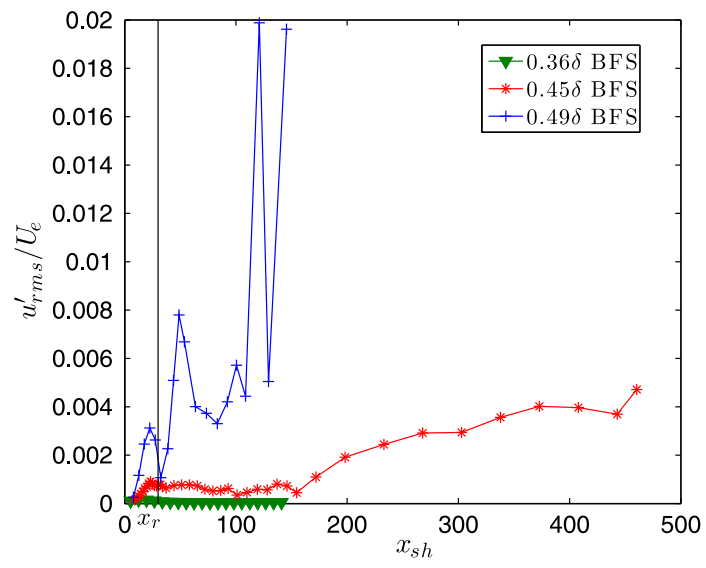

c) $\mathbf{H}$ band

Fig. 9 Peak unsteady disturbance growth along a streamline: small DREs.

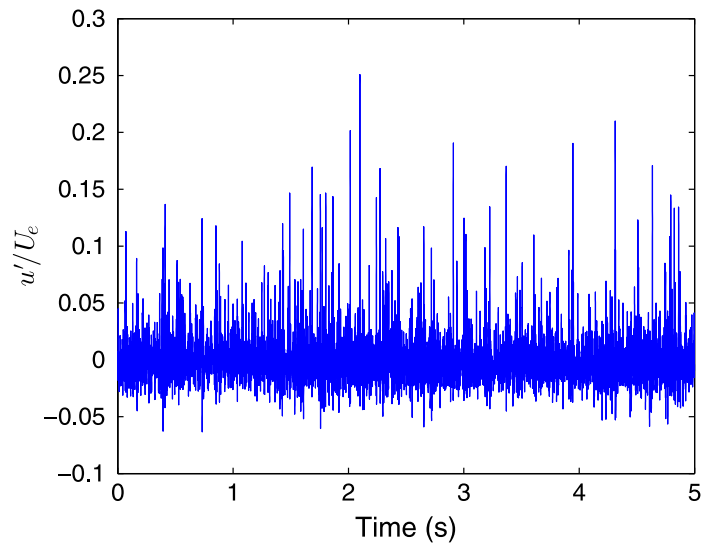

a) Time trace example

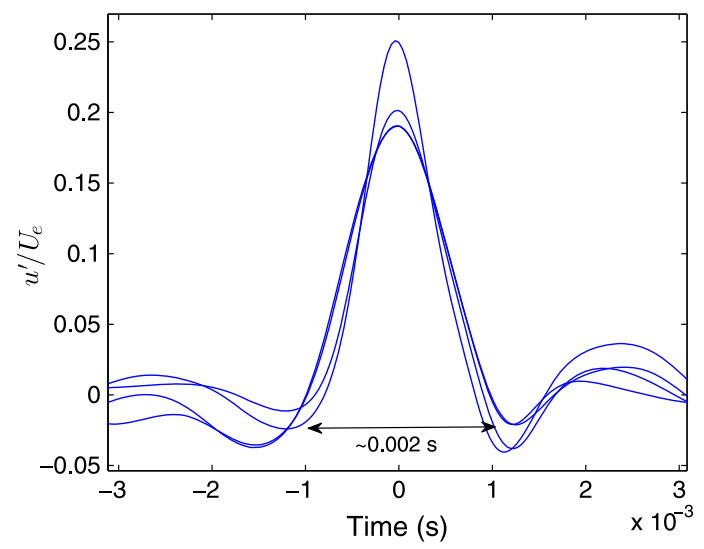

b) Several single spikes, translated arbitrarily in time to be centered at zero

Fig. 10 Time trace from a point near the wall $(y \approx 0.2 \mathrm{~mm})$ showing numerous positive spikes. 


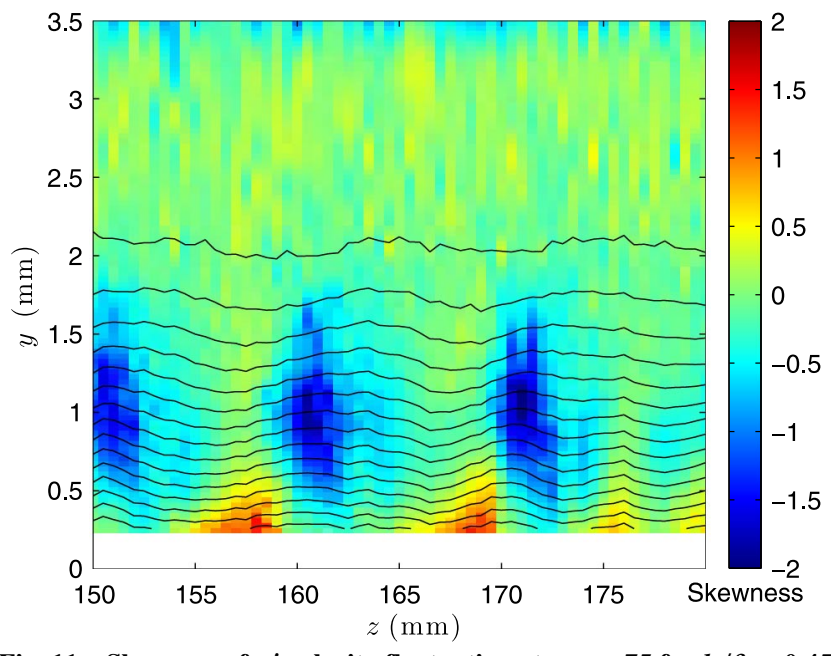

Fig. 11 Skewness of $u^{\prime}$ velocity fluctuation at $x_{\mathrm{sh}}=75$ for $h / \delta=0.45$ with large DREs. Solid black lines are mean flow contours.

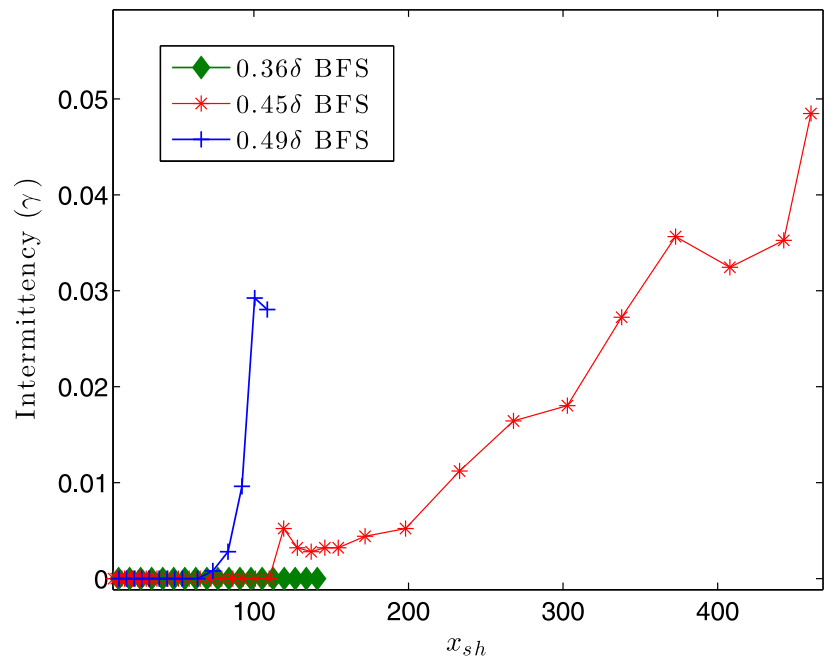

Fig. 12 Intermittency vs $x_{\text {sh }}$ with small DREs for all three step heights.

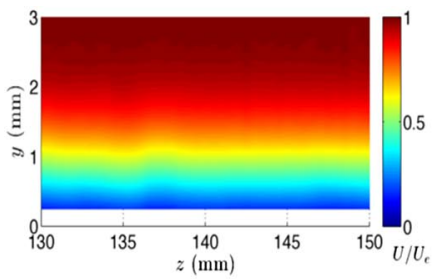

a) Mean flow

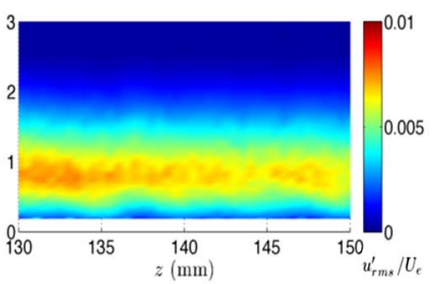

b) L band

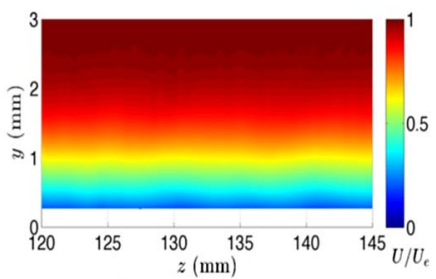

e) Mean flow

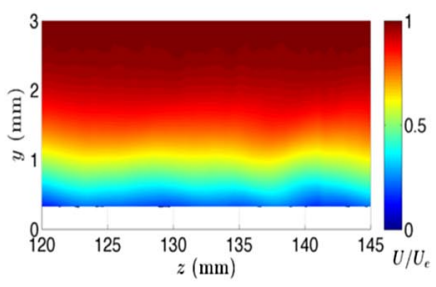

i) Mean flow

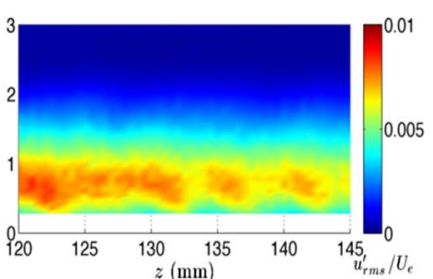

f) L band

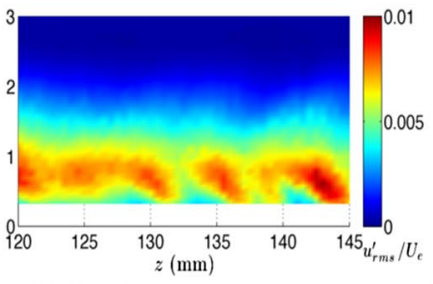

j) L band height, the unsteady disturbances still grow, but then they peak at lower amplitudes and decay. The intermediate step height (red lines) leads to significantly lower amplitudes for all three disturbance bands when compared to the case of the largest step height. In fact, the overall maximum of the M-band amplitude (Fig. 9b), which is achieved near $x_{\mathrm{sh}}=100$, is less than $2 \%$ of $U_{e}$. The H-band amplitude (Fig. 9c) remains below $0.1 \% U_{e}$ in amplitude until $x_{\mathrm{sh}} \approx 170$. The $\mathrm{L}$ band also levels off in amplitude at around $x_{\mathrm{sh}}=150$ at approximately $1 \% U_{e}$. Despite the relatively low amplitude of the unsteady disturbances, one still observes an onset of laminar flow breakdown at the intermediate step height, but (as will be discussed later in this section) the breakdown process is much slower than that observed for the largest step height. At the intermediate step height $(h / \delta=0.45)$, the disturbance growth upstream of $x_{\mathrm{sh}} \approx 150$ to 200 is due to growth of the instabilities, whereas downstream of this location, the energy growth is due to spectral broadening caused by the appearance of largeamplitude spikes and the beginning of the breakdown process. The last three points in the figures indicate a strange behavior for the largest step height (blue lines) such that the disturbance amplitudes suddenly increase drastically, decrease, and then increase again. The cause behind these large and abrupt variations is presently unclear. However, the larger-amplitude values are out of character and are believed to be spurious results. For this reason and for clarity of presentation, the results presented throughout the rest of the paper omit the last three points for this case $(h / \delta=0.49$; small DREs).

For the large and intermediate step heights, the $\mathrm{H}$ band (Fig. 9c) undergoes a short region of growth in the separated region followed by a short region of decay. The large step height also results in another region of growth just downstream of reattachment $x_{r}$. The disturbance peaks in amplitude approximately 50 step heights downstream of the step and subsequently decays. In the largest step height case, this second region of growth is quite significant; however, the H-band amplitude does not even reach $1 \% U_{e}$ before the disturbance decays again. This second region of growth is accompanied by a drastic change in the wave angle of the $\mathrm{H}$ band just downstream of reattachment. The disturbance changes from a nearly streamwisetraveling wave to a nearly spanwise-traveling wave, similar to the $\mathrm{L}$ band. Due to this change in behavior, the fact that the growth occurs downstream of reattachment (which would not be expected for a simple shear-layer instability) and there is an observed increase in bicoherence between the $\mathrm{L}$ and $\mathrm{H}$ bands (discussed by Eppink [20]),

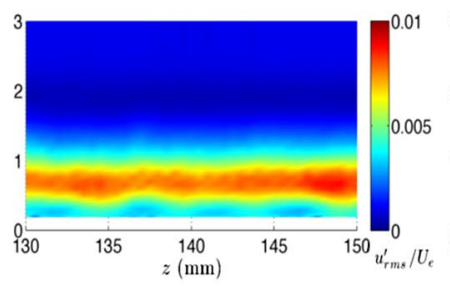

c) $M$ band

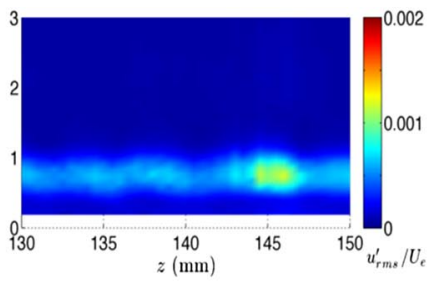

d) $\mathrm{H}$ band

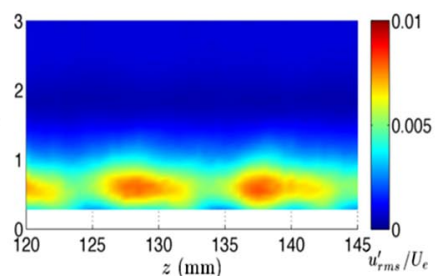

g) $\mathrm{M}$ band

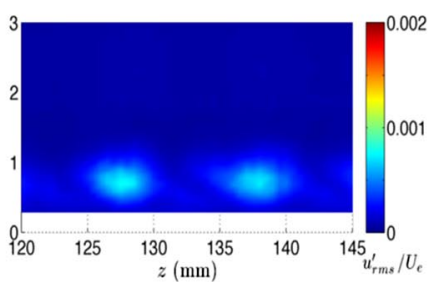

h) H band

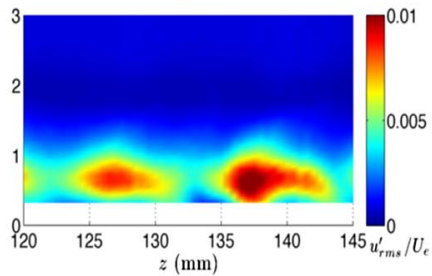

k) $\mathrm{M}$ band

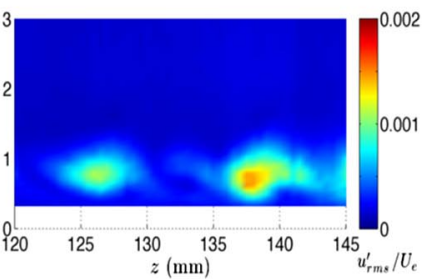

l) $\mathbf{H}$ band

Fig. 13 Contours of mean flow and rms velocity for large step height at $x_{\mathrm{sh}} \approx 30$ for no (top row), small (middle row), and large (bottom row) DREs. 
this second region of growth of the $\mathrm{H}$ band is believed to be due to nonlinear interactions with the $\mathrm{L}$ band.

The lowest step height results in amplitude growth in the $\mathrm{M}$ and $\mathrm{H}$ bands; as expected, the peak amplitudes are much lower than those at the two larger step heights. The M band peaks at an amplitude of $0.3 \%$ of $U_{e}$, whereas the $\mathrm{H}$ band grows just slightly above the noise floor, peaking at $0.02 \% U_{e}$ at 12 step heights downstream of the step before decaying. There is no visible growth in the L-band frequency range for this case.

Breakdown for both of the larger step height cases was preceded by the appearance of large spikes in velocity. A time trace showing this phenomenon is shown in Fig. 10 , along with a figure zoomed in on several positive spikes that have been translated in time to be centered at zero. This figure is included to show that the spikes themselves are not high frequency, such as the spikes seen by Klebanoff et al. [21]

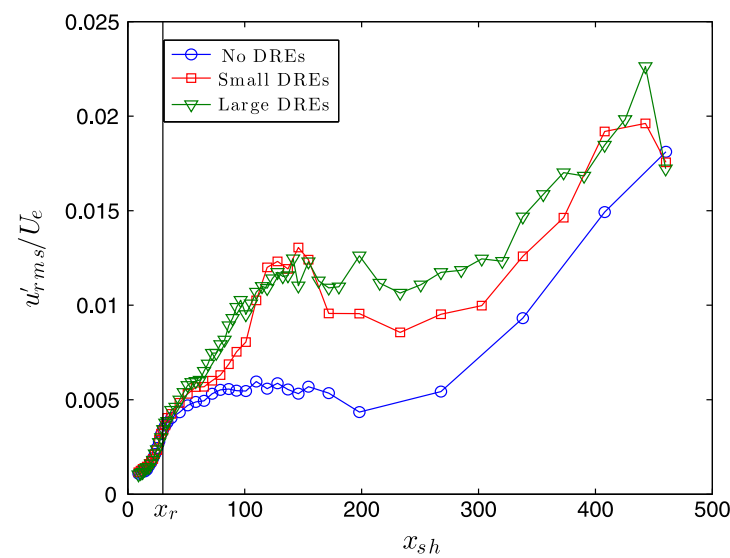

a) $L$ band, intermediate step

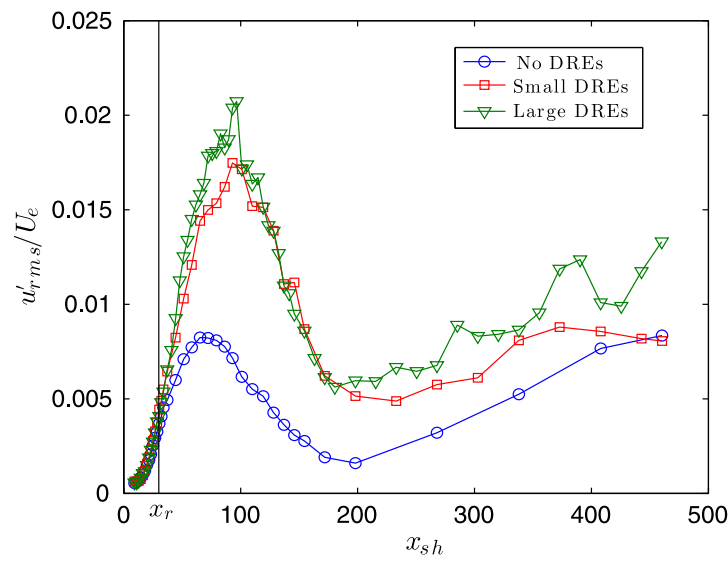

c) M band, intermediate step

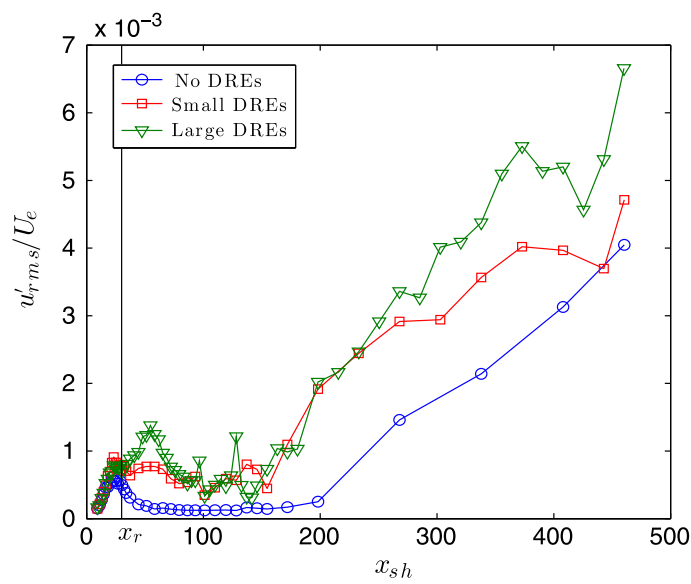

e) $\mathrm{H}$ band, intermediate step during the fundamental breakdown of TS waves on a flat plate, in which the spike frequency was an order of magnitude higher than the primary instability frequency. The spikes shown in Fig. $10 \mathrm{~b}$ all have a similar time duration of $0.002 \mathrm{~s}$, which corresponds to a frequency of about $500 \mathrm{~Hz}$.

Similar to Klebanoff et al.'s [21] results, positive and negative spikes occurred at different spanwise and wall-normal locations and seemed to be spatially related to the spanwise modulation of the unsteady disturbances, and thus to the modulation of the mean flow, as illustrated in Fig. 11. The skewness is plotted in this figure as a way of showing the spatial locations of the positive and negative spikes. The positive spikes (associated with a positive skewness factor) typically appear near the wall (as in Fig. 10a), whereas the negative spikes occur off of the wall and are offset in the spanwise direction from the locations of the positive spikes. Klebanoff et al. [21]

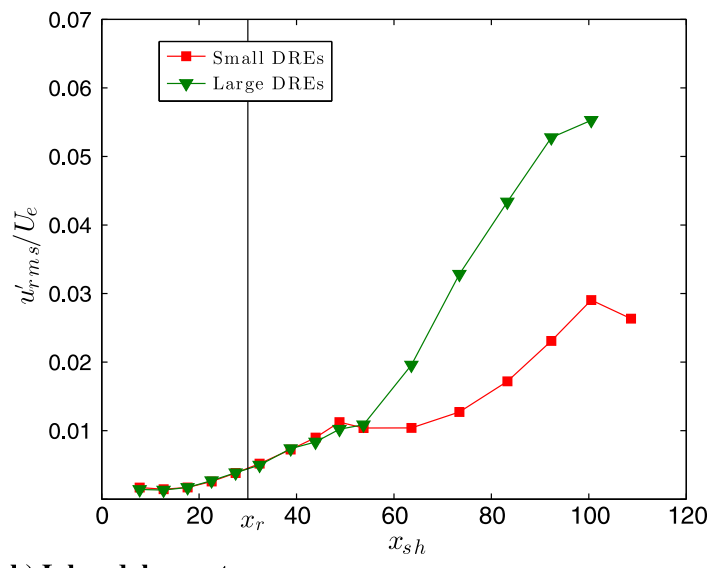

b) $L$ band, large step

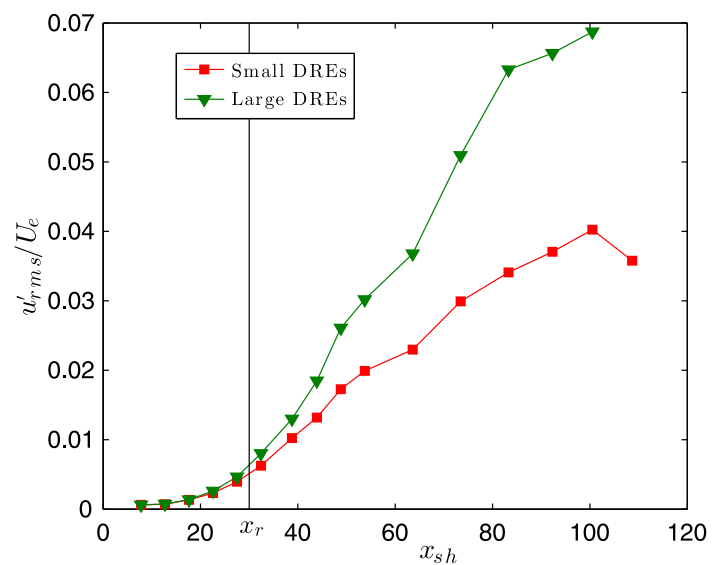

d) $M$ band, large step

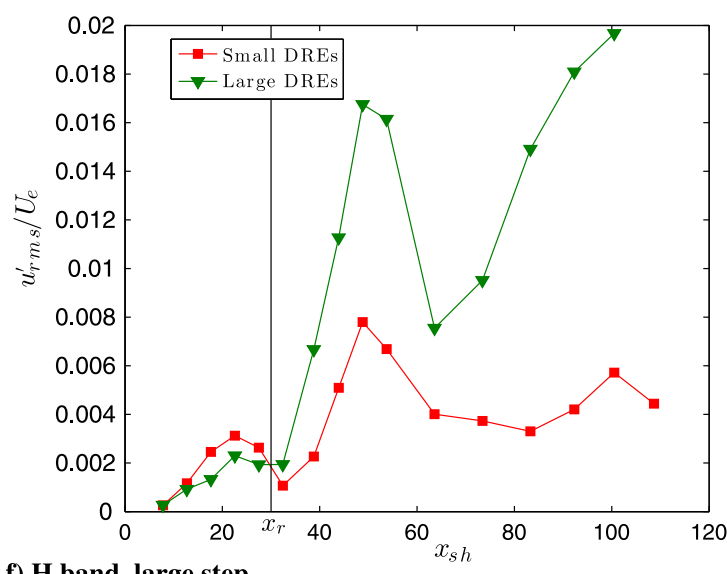

f) $\mathrm{H}$ band, large step

Fig. $14 u_{\mathrm{rms}}^{\prime}$ vs $x_{\text {sh }}$ for intermediate and large step heights. 
conjectured that the spikes that they observed were due to the shedding of hairpin eddies. It is not clear whether these spikes were related to a similar mechanism, but they are now believed to be due to a breakdown mechanism that was caused by interactions of the unsteady disturbances (see the work of Eppink and Yao [16]).

Eventually, the spikes become very large in peak amplitude $(\geq 0.2$ to $0.3 U_{e}$ ) and begin to cause the appearance of high-frequency fluctuations indicative of breakdown. To illustrate the progression of the breakdown process, a plot of intermittency vs $x_{\mathrm{sh}}$ is shown in Fig. 12. The intermittency is computed for each time trace by first high-pass filtering the data at a cutoff frequency of $6250 \mathrm{~Hz}$. The high-pass filtered data are then analyzed by computing the rms values throughout the time trace using a moving window of approximately $0.01 \mathrm{~s}$ in duration. Additionally, a "laminar flow" rms value is estimated from the same time trace by finding the minimum rms (from the previously computed rms values) in a larger moving window with a size of approximately $0.2 \mathrm{~s}$ and averaging these minimum values across the whole time trace. This procedure is used to exclude any of the turbulent sections of the time trace so that we can obtain a good baseline rms to use for the threshold calculation. Because the intermittency values remain low throughout the measurement region (usually much less then 0.35 ), this procedure works well. Based on a manual comparison of the time trace and the computed rms values throughout the time trace, we choose an intermittency threshold of five times the rms fluctuation of the laminar flow value of the computed rms. The intermittency for the time trace is then computed as the fraction of time that the rms exceeds this threshold value.

The maximum intermittency across the boundary layer at the selected spanwise station is plotted in Fig. 12. In the case of the largest step height, the intermittency begins to increase rather rapidly near $x_{\mathrm{sh}} \approx 90$. For the intermediate step height, the intermittency rises more gradually, beginning farther downstream at $x_{\mathrm{sh}} \approx 120$. Recall that Fig. 9 shows a prominent growth in all three bands of disturbance frequencies in the region of $x_{\mathrm{sh}}=150$ to 200 , indicating that the spectral broadening occurs just downstream of the rise in intermittency near $x_{\text {sh }}=120$. Thus, this observation is consistent with the hypothesis that the growth in the different instability bands downstream of this location corresponds to spectral broadening and the beginning of breakdown rather than modal growth. The main difference between the large and intermediate step heights is the length of the breakdown region. The spikes occurring downstream of the intermediate step are extremely intermittent, and thus the transition region is much longer than that for the larger step.

\section{Effect of Stationary Crossflow Amplitude on Breakdown}

As was mentioned briefly in the previous section, the initial stationary crossflow amplitude was found to have an effect on the amplitude of the unsteady disturbances. This effect is clearly seen in Fig. 13 , in which bandpass-filtered $u_{\mathrm{rms}}^{\prime}$ contours are shown at $x_{\mathrm{sh}} \approx \overline{30}$ for all three DRE configurations downstream of the large
$(0.49 \delta)$ step. Note that the DRE configuration dictates the initial amplitude of stationary crossflow modes in a given case. The unsteady instabilities become more modulated as the stationary crossflow amplitude is increased. The peak unsteady amplitudes in each frequency band also increase with initial stationary crossflow amplitude.

The influence of the DRE configuration on the amplitude evolution for each of the three frequency bands is plotted in Figs. 14a, 14c, and $\underline{14 \mathrm{e}}$, respectively, for the intermediate step height and Figs. $1 \overline{4 \mathrm{~b}}, 14 \mathrm{~d}$, and $14 \mathrm{f}$ for the largest step height. In each figure, the growth of the unsteady disturbances increases as the stationary crossflow initial amplitude is increased from no DREs, to small DREs, to large DREs, respectively. The effect of the initial stationary crossflow amplitude on the amplification of the traveling disturbances is particularly evident in the region downstream of the reattachment location. This section briefly explores how the breakdown process associated with spikes in the velocity signal and the resulting evolution in signal intermittency are influenced by the DRE configuration.

The evolution of intermittency vs $x_{\mathrm{sh}}$ is shown in Fig. 15 for the intermediate and large step cases. Note the different y-axis scales in these figures. The increase in the stationary crossflow initial amplitude clearly causes a more rapid rise in intermittency for both step cases. Thus, the increase in the number and amplitude of the spikes caused by the increase in stationary crossflow amplitude leads to earlier and faster breakdown. The difference in intermittency evolution across the different DRE configurations is much more drastic for the larger step height than for the intermediate step height. Note that the transition locations listed in Table $\underline{1}$ are acquired from the naphthalene flow visualization and occur much farther downstream than anything shown on these plots. Because the sublimating chemical will be expected to show the mean shear behavior of the flow, it is expected that the intermittency will need to reach large values before the mean profiles become distorted enough for the naphthalene to show a change in the transition front.

\section{Conclusions}

Previously reported measurements related to the effects of a backward-facing step on laminar-turbulent transition in a sweptwing boundary layer were extended in terms of both measurement detail and the range of step heights in order to gain new insights into the transition mechanisms involved. Three different heights of a backward-facing step were studied, ranging from 36 to $49 \%$ of the local unperturbed boundary-layer thickness, as well as three different stationary crossflow initial amplitudes. The largest step height resulted in premature transition occurring shortly downstream of the step, and the transition location moved closer to the step as the stationary crossflow initial amplitude was increased. The two smaller step heights did not result in an upstream movement of the transition front. However, in the case of the intermediate step height, unsteady measurements revealed a long and intermittent breakdown region initiated by the step.
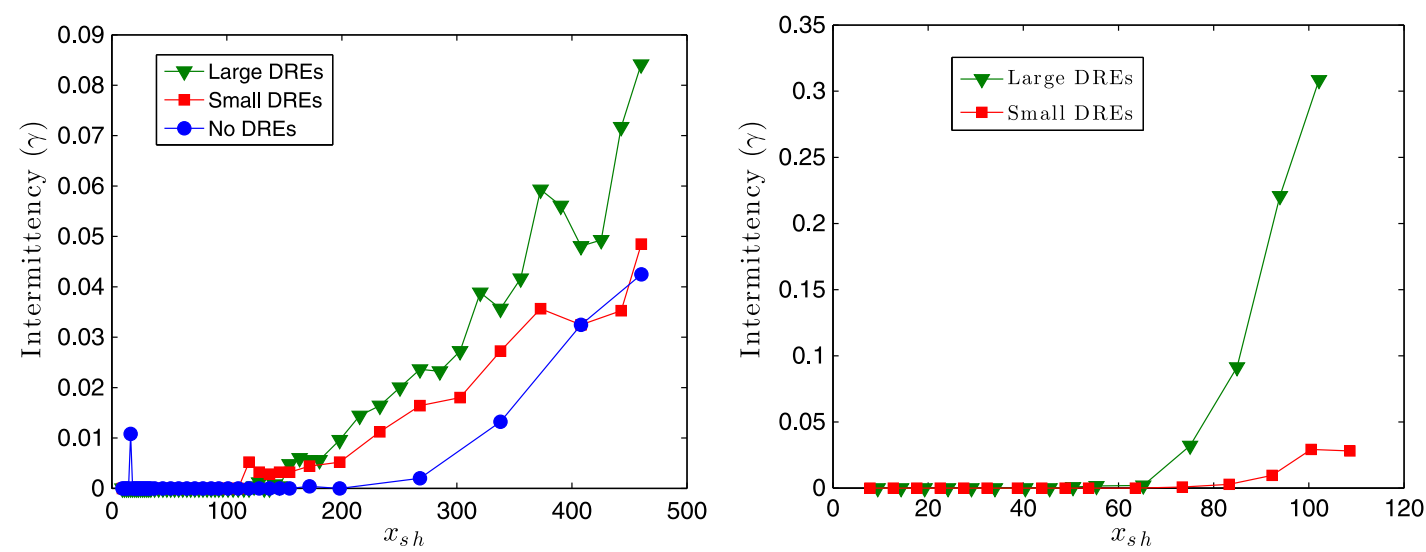

a) Intermediate step

b) Large step

Fig. 15 Intermittency vs $x_{\text {sh }}$ for the intermediate and large step cases. 
Both steady and unsteady hot-wire data were acquired to study the growth of instabilities downstream of the step. Streamwise amplification of stationary crossflow disturbances was investigated for the dominant wave number of the stationary crossflow instability, and the results were compared with analogous measurements for the baseline, i.e., the zero-step case. The stationary crossflow mode was locally affected by the step but did not directly lead to transition at the largest step height that corresponded to a barely postcritical surface irregularity. However, stationary crossflow was still found to be important in the transition process with the step present. Even at very low amplitudes $\left(1-2 \% U_{e}\right)$, spanwise modulation of unsteady disturbances by the stationary modes could lead to significant three-dimensionality, which is known to be important for the breakdown process.

The growth of the unsteady disturbances was also studied by tracking the streamwise evolution of the peak disturbance amplitude. The amplitudes decreased with decreasing step height and with decreasing initial amplitude of stationary crossflow disturbances. To gain further insights into the nature of the transition process, the disturbance evolution was monitored across three different bands of frequencies, namely, the $\mathrm{L}$ band ( $f=80$ to $200 \mathrm{~Hz}$ ), the $\mathrm{M}$ band ( $f=200$ to $800 \mathrm{~Hz}$ ), and the $\mathrm{H}$ band ( $f=800$ to $1500 \mathrm{~Hz}$ ). At the lowest step height $(h / \delta=0.36)$, the L band was not observed. At the intermediate step height $(h / \delta=0.45)$, the amplitudes of the $\mathrm{L}$ and $\mathrm{M}$ bands peaked between 1 and $2 \% U_{e}$ before decaying, and the $\mathrm{H}$ band was an order of magnitude lower in amplitude. In the largest step height case, the $\mathrm{L}$ and $\mathrm{M}$ bands grew much faster than in the smaller step cases, reaching amplitudes of approximately $7 \% U_{e}$ by $x_{\mathrm{sh}} \approx 100$. The $\mathrm{H}$ band grew initially in the separated region, and then it decayed and grew again downstream of reattachment, peaking at around $x_{\mathrm{sh}}=50$. Whereas the observation that the unsteady disturbance amplitude decreased with decreasing step height was to be expected, the finding that the $\mathrm{L}$ band was entirely absent for the smallest step height was difficult to explain. Another unexpected finding from the experimental measurements involved the occurrence of laminar flow breakdown (i.e., the appearance of intermittent high-amplitude disturbances) at the intermediate step case, even though all three disturbance bands remained low in amplitude.

Breakdown of the laminar boundary layer downstream of the step was preceded by the appearance of large spikes in the velocity for the two cases with the larger step heights. No spikes were observed for the smallest step height. The spikes occurred locally in both time and space, with positive spikes occurring near the wall and the negative spikes occurring away from the wall and offset in spanwise location with respect to the positive spikes. Depending on the step height and initial amplitude of the stationary crossflow disturbance, the spikes increased in number and amplitude, starting downstream of the reattachment location. Eventually, the spikes reached sufficiently large amplitudes and began to induce breakdown. The spikes remained extremely intermittent for the intermediate step height ( $h / \delta=0.45$ ). Thus, if one considers the beginning of transition to be the location at which a single spike started to exhibit high-frequency breakdown (spectral broadening), then the transition region was much longer for the intermediate step height as compared with that for the largest step height $(h / \delta=0.49)$.

The following important conclusions can be drawn from this work. In this scenario of a backward-facing step downstream of the neutral point, the sudden, upstream movement in transition location when the step height exceeds the critical step height is related to a change in the transition mechanism with respect to the baseline case of a smooth airfoil surface. However, the altered transition mechanism does not necessarily bypass the growth of primary disturbances. It merely involves a modified role of the stationary crossflow vortices. Whereas transition in the baseline (i.e., the zero step height case) is due to highfrequency secondary instabilities of large-amplitude stationary crossflow modes, transition at slightly postcritical step heights is initiated by nonlinear interactions between moderate-amplitude stationary crossflow modes and unsteady disturbances including one or more disturbance types out of traveling crossflow modes and Tollmien-Schlichting waves in the 3-D boundary layer, as well as
Kelvin-Helmholtz instabilities of the inflectional profiles behind the step. Traditionally, a sudden movement in transition location due to the steps would have been called a bypass transition because of the unknown nature of the transition mechanism accounting for this shift. However, it is believed that the measurements presented herein provide useful insights into that previously unknown transition mechanism. The nonlinear interactions mentioned previously led to localized spikes in the velocity signal, which began to appear shortly downstream of the step and, depending on the extent of the boundarylayer modification due to the step, could persist over a broad range of distances before a fully turbulent flow developed.

A second conclusion, related to the first, is that the stationary crossflow vortices (and thus sweep) play a role in this critical behavior because the stationary vortices cause modulation and increased amplification of the unsteady disturbances. An analysis of the hot-wire measurements suggests that the interaction of the stationary crossflow vortices and the unsteady disturbances leads to the appearance of these spikes and early breakdown. The spanwise spacing of both positive and negative spikes in the velocity signal corresponds to the spanwise wavelength of the dominant stationary crossflow mode, and thus the spike locations are related to the locations of the peaks and valleys in the spanwise amplitude distribution of the unsteady disturbances (caused by the interaction with the stationary crossflow vortices). The laminar flow breakdown begins to occur when the disturbance amplitudes are still very low $\left(1-2 \% U_{e}\right)$. Further work is being performed to try to gain a better understanding of this breakdown mechanism and how it is triggered. A better understanding of what triggers transition in these sweptwing excrescence cases will allow for higher-fidelity transition prediction.

\section{Acknowledgments}

This work was performed as part of the revolutionary computational aerosciences discipline under the Transformational Tools and Technologies project of the NASA Fundamental Aeronautics Program. The authors would like to thank William T. Jones for his assistance with the ceiling contour design, Jackson Johnson for fabrication of the ceiling liner, Charlie Debro for his support of the wind-tunnel testing, and the members of the Flow Physics and Control Branch of NASA Langley Research Center for their support and many helpful discussions.

\section{References}

[1] Holmes, B., Obara, C., Martin, G., and Domack, C., "Manufacturing Tolerances for Natural Laminar Flow Airframe Surfaces," General Aviation Meeting \& Exposition, SAE Paper 850863, Wichita, KS, 1985. doi: $10.4271 / 850863$

[2] Fage, A., "The Smallest Size of a Spanwise Surface Corrugation Which Affects Boundary Layer Transition on an Aerofoil," British Aeronautical Research Council R\&M 2120, London, 1943.

[3] Carmichael, B., "Surface Waviness Criteria for Swept and Unswept Laminar Suction Wings," Norair Rept. NOR-59-438 (BLC-123), Hawthorne, CA, 1959.

[4] Sedney, R., "A Survey of the Effects of Small Protuberances on BoundaryLayer Flows," AIAA Journal, Vol. 11, No. 6, 1973, pp. 782-792. doi: $10.2514 / 3.50520$

[5] Tani, I., "Boundary-Layer Transition," Annual Review of Fluid Mechanics, Vol. 1, No. 1, 1969, pp. 169-196. doi:10.1146/annurev.fl.01.010169.001125

[6] Crouch, J., Kosorygin, V., and Ng, L., "Modeling the Effects of Steps on Boundary-Layer Transition," IUTAM Symposium on LaminarTurbulent Transition, Springer, New York, 2006, pp. 37-44.

[7] Wang, Y., and Gaster, M., "Effect of Surface Steps on Boundary Layer Transition," Experiments in Fluids, Vol. 39, No. 4, 2005, pp. 679-686. doi:10.1007/s00348-005-1011-7

[8] Perraud, J., and Seraudie, A., "Effects of Steps and Gaps on 2-D and 3-D Transition," European Congress on Computational Methods in Applied Science and Engineering, ECCOMAS, Barcelona, Spain, 2000, pp. 11-14.

[9] Masad, J., "The Critical Allowable Height of a Backward-Facing Step," AIAA Paper 1996-0780, 1996. doi: $10.2514 / 6.1996-780$ 
[10] Duncan, G., Jr., Crawford, B., and Saric, W., "Effects of Step Excrescences on Swept-Wing Transition," AIAA Paper 2013-2412, 2013.

doi: $10.2514 / 6.2013-2412$

[11] Duncan, G. T., Jr., Crawford, B. K., Tufts, M. W., Saric, W. S., and Reed, H. L., "Effects of Step Excrescences on a Swept Wing in a LowDisturbance Wind Tunnel," AIAA Paper 2014-0910, 2014. doi:10.2514/6.2014-0910

[12] Tufts, M. W., Reed, H. L., Crawford, B. K., Duncan, G. T., Jr., and Saric, W. S., "Computational Investigation of Step Excrescence Sensitivity in a Swept-Wing Boundary Layer," Journal of Aircraft, Vol. 54, No. 2, 2017, pp. $602-626$ doi:10.2514/1.C033892

[13] Crawford, B. K., Duncan, G. T., Tufts, M. W., Saric, W. S., and Reed, H. L., "Effects of Step-Excrescence Location on Swept-Wing Transition," AIAA Paper 2013-2412, 2013. doi:10.2514/6.2015-1233

[14] Eppink, J. L., Wlezien, R. W., King, R. A., and Choudhari, M., "Interaction of a Backward-Facing Step and Crossflow Instabilities in Boundary-Layer Transition," AIAA Journal, Vol. 56, No. 2, 2018, pp. 497-509. doi:10.2514/1.J056267

[15] Eppink, J., and Yao, C.-S., "Stereo Particle Image Velocimetry Measurements of Transition Downstream of a Backward-Facing Step in a Swept-Wing Boundary Layer," AIAA Paper 2017-0305, 2017. doi: $10.2514 / 6.2017-0305$
[16] Eppink, J. L., and Yao, C., "Time Resolved Stereo Particle Image Velocimetry Measurements of the Instabilities Downstream of a Backward-Facing Step in a Swept-Wing Boundary Layer," AIAA Paper 2017-4415, 2017. doi:10.2514/6.2017-4415

[17] Balakumar, P., King, R. A., and Eppink, J. L., "Effects of Forward-and Backward-Facing Steps on the Crossflow Receptivity and Stability in Supersonic Boundary Layers," AIAA Paper 2014-2639, 2014. doi:10.2514/6.2014-2639

[18] Saric, W. S., and Reshotko, E., "Review of Flow Quality Issues in Wind Tunnel Testing," AIAA Paper 1998-2613, 1998. doi:10.2514/6.1998-2613

[19] Eppink, J., Wlezien, R., King, R., and Choudhari, M., "The Interaction of A Backward-Facing Step and Crossflow Instabilities in BoundaryLayer Transition," AIAA Paper 2015-0273, 2015. doi:10.2514/6.2015-0273

[20] Eppink, J., "The Interaction of Crossflow Instabilities and a BackwardFacing Step in Swept Boundary Layer Transition," Ph.D. Thesis, Tufts Univ., Medford, MA, 2014.

[21] Klebanoff, P., Tidstrom, K., and Sargent, L., "The Three-Dimensional Nature of Boundary-Layer Instability," Journal of Fluid Mechanics, Vol. 12, No. 1, 1962, pp. 1-34. doi: $10.1017 / \mathrm{S} 0022112062000014$

B. Ganapathisubramani Associate Editor 